\title{
Impacto de los nuevos criterios para diagnóstico y tratamiento de la hipertensión arterial sistémica sugeridos por la American College of Cardiology/American Heart Association
}

\author{
Martín Rosas-Peralta y Gabriela Borrayo-Sánchez \\ Instituto Mexicano del Seguro Social, Programa "A todo Corazón-Código Infarto", Ciudad de México, México
}

El nuevo informe del Grupo de Trabajo del American College of Cardiology y la American Heart Association (ACC/AHA) sobre las guías de práctica clínica para la presión arterial alta en adultos se publicó en línea antes del 13 de noviembre de 2017. ${ }^{1}$ La nueva recomendación estadounidense se centró en el criterio para definir la hipertensión: $\geq 130 / 80 \mathrm{~mm} \mathrm{Hg}$ se considera el nuevo punto de corte (Tabla 1), lo cual no es nuevo si consideramos que la evidencia acumulada en las dos últimas décadas derivó en considerar 140/90 mm Hg como referencia para iniciar acciones médicas.

Ya con los criterios convencionales (> 140/0 mm Hg) existían grandes retos para el diagnóstico, tratamiento y seguimiento del paciente portador de hipertensión. La cifra estimada en México en 2017 era de $22.2 \mathrm{mi}$ llones de adultos hipertensos, de los cuales cerca de la mitad estaba sin diagnóstico y solo $60 \%$ de los diagnosticados estaba en tratamiento farmacológico y apenas poco más de la mitad con cifras de buen control.

El problema de la hipertensión arterial debe ser reconocido en nuestro país como un problema social y de salud pública, pues la prevalencia es muy alta y se trata del principal factor de riesgo cardiovascular, que contribuye con $1.5 \%$ de muertes directamente relacionadas y colabora al desarrollo de la aterosclerosis, causa primaria de mortalidad en México y el mundo.

En México, la prevalencia de la hipertensión arterial en los adultos mayores de 20 años había sido de aproximadamente de $30 \pm 2 \%$, según las encuestas nacionales de salud de 2000, 2006 y $2012 .^{2}$ Sin embargo, la Encuesta Nacional de Salud a Medio Camino de $2016^{3}$ indicó una prevalencia de $25.5 \%$, disminución drástica derivada de aspectos metodológicos. El significado de lo anterior cobra relevancia si se toma en cuenta que la hipertensión arterial constituye el factor de riesgo cardiovascular más común asociado con la enfermedad de la arteria coronaria y la muerte cardiovascular. ${ }^{4}$

El problema en términos de reducción de riesgo cardiovascular es complejo y difícil de abordar. Desafortunadamente, la hipertensión arterial sistémica no es una entidad nosológica aislada, cohabita frecuentemente con diabetes, dislipidemia y obesidad. Ya sea en pares o como síndrome metabólico, estos factores de riesgo tienen vías fisiopatológicas comunes y se sabe que uno potencia al otro respecto a la progresión del daño tisular. Por lo tanto, una estrategia de salud nacional requiere una visión integral y global de la hipertensión arterial sistémica. Consideramos que en el mundo se han realizado numerosos esfuerzos y que cada país ha comprendido que sus estrategias deben ser únicas y especiales conforme su situación sociodemográfica y multifactorial.

En nuestro país, el Instituto Mexicano del Seguro Social han desarrollado diversas estrategias para mejorar la prevención como acción clave para enfrentar las enfermedades crónicas no contagiosas, incluida la hipertensión. La guía actualizada del ACC/AHA es una oportunidad extraordinaria para reforzar los programas preventivos para el control de la presión arterial alta. En esta editorial analizamos la situación epidemiológica en México y sus posibles consecuencias de los nuevos criterios para el diagnóstico de hipertensión. Las principales estrategias actuales que se aplican en el Instituto Mexicano del Seguro Social para enfrentar los factores de riesgo cardiovascular están dirigidas a la prevención. Esta institución se
Gac Med Mex. 2018;154:633-637 Disponible en PubMed www.gacetamedicademexico.com 
Tabla 1. Nueva clasificación de hipertensión arterial

\begin{tabular}{lc}
\hline Normal & $<120 / 80 \mathrm{~mm} \mathrm{Hg}$ \\
Elevada & $120-129 /<80 \mathrm{~mm} \mathrm{Hg}$ \\
Etapa 1 & $130-139 / 80-89 \mathrm{~mm} \mathrm{Hg}$ \\
Etapa 2 & Por lo menos $140 / 90 \mathrm{~mm} \mathrm{Hg}$ \\
Crisis hipertensiva & $>180 / 120 \mathrm{mg} \mathrm{Hg}$ \\
\hline AHA/ACC, noviembre de 2017 &
\end{tabular}

esfuerza por atender situaciones como el cambio en los criterios diagnósticos en la hipertensión y nuevos modelos preventivos están en progreso.

\section{El panorama en diversos países}

\section{China $^{5}$}

China ha recibido con preocupación los nuevos lineamientos, ya que si bien el incremento de la prevalencia en porcentaje aparentemente no es mucho, en números absolutos de millones de pacientes resulta muy oneroso. A pesar de ello, en ese país se considera que el cambio de los criterios resulta beneficioso para la prevención cardiovascular. El concepto de tratamiento temprano concuerda con el enfoque del curso de vida propuesto en la llamada a la acción china de la Comisión de Hipertensión publicado en 2016 en Lancet. $^{3}$

Para juzgar la utilidad y conveniencia del nuevo umbral, tiene que considerarse su influencia en las perspectivas a corto y largo plazo. Este último, por supuesto, requiere tiempo y solo puede ser juzgado históricamente según la incidencia de las complicaciones cardiovasculares de la hipertensión arterial. Sin embargo, se pueden obtener conclusiones a corto plazo si el cambio mejora el control de la presión arterial sin aumentar sustancialmente el costo, la carga de trabajo y efectos secundarios del tratamiento.

Al aplicar el umbral de $130 / 80 \mathrm{~mm} \mathrm{Hg}$ a los datos más recientes de la encuesta nacional de China, la prevalencia de la hipertensión arterial aumentaría de aproximadamente 25 a $50 \%$, si bien se afirma que el incremento en el número de pacientes que requerirán tratamiento es marginal (Figura 1). De hecho, en nuestros estudios en población general y en una población de edad avanzada el aumento fue de 2 a $5.5 \%$, respectivamente. Si menos de $50 \%$ de la tasa general de la población que se conoce con hipertensión en China se tuviera en cuenta, el aumento en el número de pacientes hipertensos tratados sería

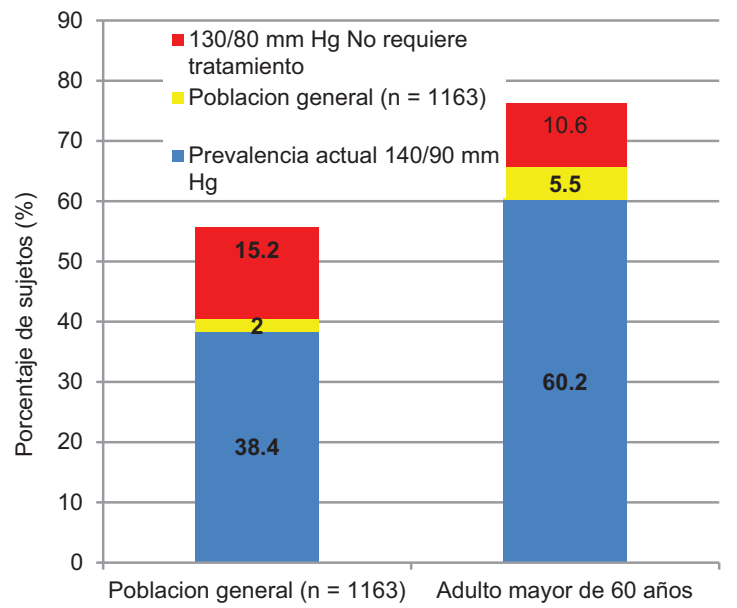

Figura 1. Distribución de población hipertensa con el criterio tradicional (140/90 $\mathrm{mm} \mathrm{Hg}$ ) y el impacto con el nuevo criterio de 130/80 mm Hg, En el adulto mayor la alta prevalencia de hipertensión minimiza el impacto, pero en México la población hipertensa tiene menos de 54 años.

insignificante. No obstante, este supuesto debe medirse con cautela en la práctica.

\section{Japón ${ }^{6}$}

Desde el punto de vista de la salud cardiovascular, una detección más temprana de la hipertensión etapa 1 con el nuevo umbral diagnóstico (130-139/80-89 mm Hg) -con un enfoque de precaución de casos no diagnosticados de presión arterial alta (sistólica de 120-129 mm Hg y diastólica < 80 mm Hg) - sería importante para minimizar el daño vascular desde edades tempranas de la vida (Figura 2). En épocas posteriores de la vida, niveles más altos de presión están asociados con la mayor variabilidad de presión arterial, lo que podría precipitar más eventos cardiovasculares, especialmente en pacientes hipertensos con alto riesgo (con diabetes, dislipidemia y obesidad). Los japoneses hacen un análisis interesante de la presión sistólica (la más común en ese país) y sus implicaciones, en el que determinan la importancia de la medición domiciliaria de la presión arterial.

\section{India ${ }^{7}$}

India, con una población de 1,320 billones, está experimentando un aumento de la enfermedad cardiovascular, principalmente por hipertensión no controlada. La prevalencia actual de la hipertensión (con base en el umbral $\geq 140 / 90 \mathrm{~mm} \mathrm{Hg}$ ) en ese país se estima en $28.9 \%^{3}$ La presión arterial está controlada en solo aproximadamente $25 \%$ de los pacientes tratados, 


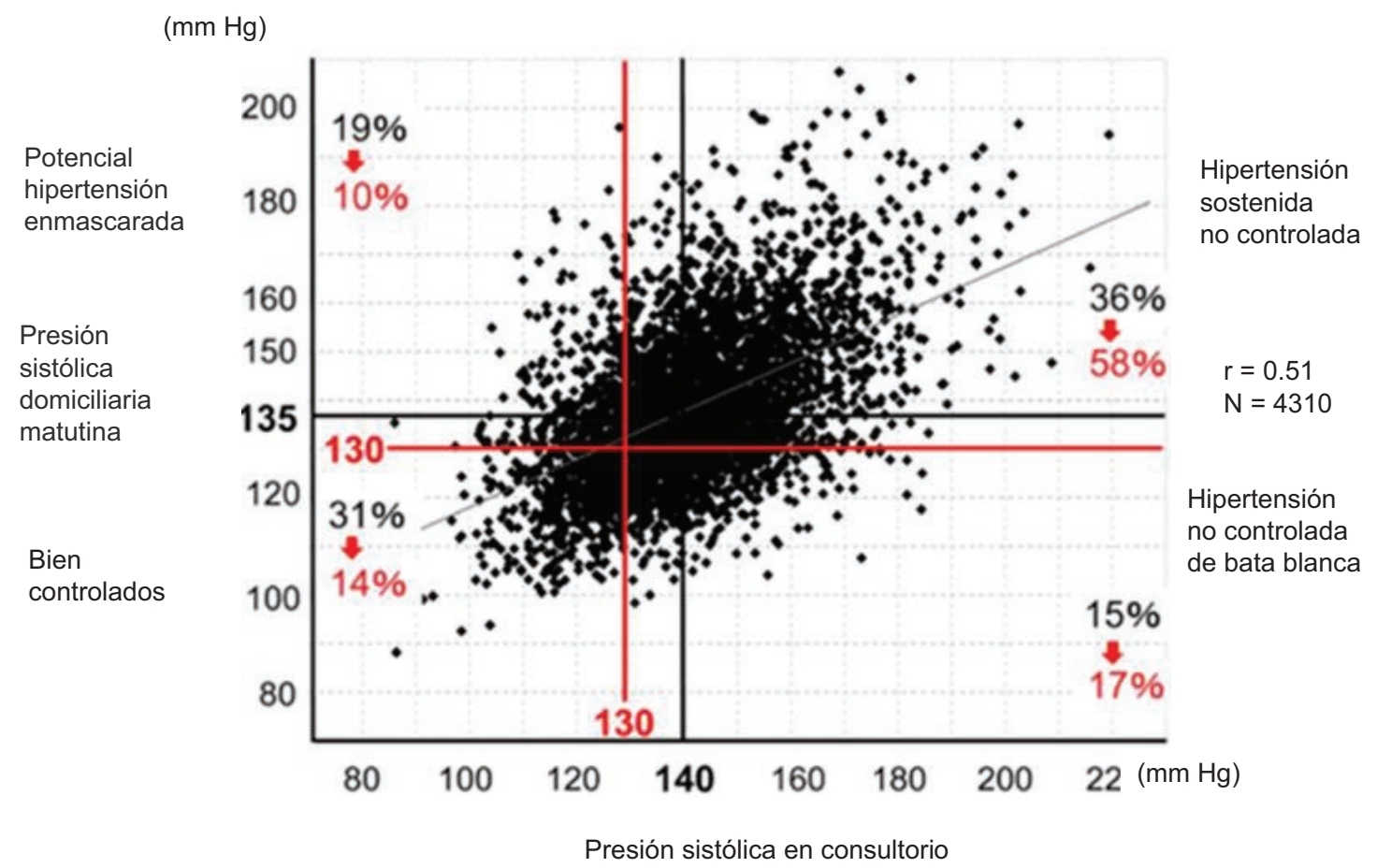

Figura 2. Impacto que tendrá el nuevo criterio sobre la presión sistólica y cómo se modifican la tasa de hipertensión genuina, la de bata blanca y la enmascarada, factor importante para tener en cuenta.

cifra que se desplomará si se incluye la nueva definición de la hipertensión arterial.

A diferencia del mundo occidental, en la India una mezcla de factores sociales, culturales y económicos hará extremadamente difícil manejar la hipertensión en la comunidad con los nuevos umbrales más bajos. Este país aún no está listo para utilizar mediciones a gran escala fuera del consultorio. Las características diferenciales del servicio médico serán un gran obstáculo para la rápida adopción de los nuevos parámetros en la hipertensión arterial.

\section{Canadá ${ }^{8}$}

La nueva categoría de presión arterial elevada y las recomendaciones de modificación de estilo de vida están destinadas a prevenir el desarrollo de la hipertensión arterial. La definición de hipertensión como presión arterial $\geq 130 / 80 \mathrm{~mm} \mathrm{Hg}$ se asemeja a lo que se sugirió en 2016 en Canadá después del SPRINT, usando además la presión media de la aorta. La nueva definición tiene sentido conforme varios metaanálisis que demostraron que disminuir el umbral de la presión arterial a $<140 / 90 \mathrm{~mm} \mathrm{Hg}$ dio lugar a mejores resultados. Además, los pacientes que se reclutaron en SPRINT con presión sistólica $\leq 132 \mathrm{~mm} \mathrm{Hg}$ se beneficiaron más que cuando la presión arterial sistólica disminuyó a $<120$ mm Hg. Con base en las estimaciones de población derivadas de la encuesta de salud nacional y examen de nutrición que abarcó de 2011 a 2014, la prevalencia de la hipertensión aumentará de 32 a $46 \%$ debido a los individuos hipertensos recién identificados como tal con los nuevos criterios. Sin embargo, muchos de ellos pertenecen a la etapa 1 de hipertensión y no son necesariamente candidatos a tratamiento farmacológico.

Se sabe que el riesgo cardiovascular comienza desde 120/80 mm Hg. No obstante, la presión arterial alta es un proceso continuo y los puntos de corte para clasificar y hacer un diagnóstico de hipertensión se deben tener en cuenta. En otras palabras, en los pacientes con diabetes y presión arterial de $135 / 83 \mathrm{~mm} \mathrm{Hg}$ con evidencia de lesión del órgano blanco (es decir, microalbuminuria) debe considerarse tratamiento farmacológico, además de los cambios en el estilo de vida. Por otro lado, en los pacientes con el mismo nivel de presión arterial pero sin evidencia de lesión del órgano blanco y solo sobrepeso podrían ser suficientes los cambios en el peso y estilo de vida.

\section{Impacto de los nuevos criterios en México}

En México, por ejemplo, a mediados de 2017 existían alrededor de 128 millones de personas, $65 \%$ (76 millones) 
correspondía a población mayor de 20 años. La prehipertensión, también llamada presión sanguínea límite, se consideró en el informe anterior del Octavo Joint National Committee y se informó que su prevalencia era de aproximadamente 25 a $30 \%$ en la población adulta.

En México, la prevalencia de prehipertensión era de $37.5 \%$ (intervalo de confianza [IC] de $95 \%=36.0-39.0$ ), $46.7 \%$ correspondió a hombres (IC del $95 \%=44.1-49.4$ ) y $33.2 \%$ a mujeres (IC $95 \%=31.5-5.0) .{ }^{9}$ Esta situación es dramática debido a que $37 \%$ de la prehipertensión en México representa alrededor de $\mathbf{3 0 . 9}$ millones de población adulta. Con la prevalencia estimada en $30 \%$ conforme al criterio de 140/90 mm Hg, alrededor de 22.8 millones corresponde al diagnóstico de hipertensión, pero con los nuevos criterios de la AHA la población con hipertensión será al menos del doble. En otras palabras, necesitaremos programas de prevención y tratamiento para alrededor de 53 millones de adultos en México. Esta es una alerta epidemiológica real que requerirá nuevas estrategias para enfrentar un viejo problema con nuevos desafíos diagnóstico-terapéuticos.

Si bien el enfoque de la ACC/AHA ${ }^{1}$ busca abordar con mayor énfasis las acciones preventivas y no farmacológicas para tratar las implicaciones de la hipertensión real en países como México, representa un importante desafío para la salud pública. La necesidad de establecer una posición es clara y necesaria.

\section{Estadio 1 de los nuevos criterios en México: ¿quiénes son candidatos a tratamiento farmacológico?}

Un aspecto relevante es que a diferencia de otros países, la hipertensión arterial se presenta con mayor frecuencia en sujetos menores de 54 años. Nuestras estadísticas de pacientes portadores de diabetes, dislipidemia y obesidad hacen que la población hipertensa se ubique en población con probabilidad más elevada de riesgo cardiovascular. Por lo anterior, debe individualizarse cada caso y no debe considerarse que el paciente en estadio I solo requiere tratamiento no farmacológico; por el contrario, debe intensificarse la búsqueda de daño a órgano blanco como retinopatía o microalbuminuria o datos de hipertrofia ventricular, que obligan a tratamiento farmacológico.

\section{Acciones actuales en México}

La transición epidemiológica de las enfermedades en México se ha reconocido desde finales del siglo pasado. Las encuestas nacionales de salud permitieron al gobierno de nuestro país establecer políticas de salud dirigidas específicamente a enfrentar esta nueva pandemia. A principios de este siglo XXI surgieron programas integrados de salud como PREVENIMSS, reconocidos como una estrategia para la prestación de servicios que tiene como propósito general la provisión sistemática y ordenada de acciones relacionadas, tales como promoción de la salud, vigilancia nutricional, prevención, detección y control de enfermedades y salud reproductiva, que estimulan la cultura del autocuidado. Actualmente los resultados de esta estrategia son muy relevantes.

Sin embargo, a pesar de la mejora en la detección oportuna de las enfermedades crónicas, el gran desafío es lograr cifras aún mayores de pacientes tratados y controlados, para evitar la generación de complicaciones tardías como infarto del miocardio e infarto cerebral, así como enfermedad arterial periférica, insuficiencia renal y ceguera secundaria a retinopatía hipertensiva y diabética. Los sistemas de capacitación en línea han permitido lograr una mayor cobertura y buscar estandarizar el conocimiento y las acciones directas en el campo operativo.

La alerta constante acerca del control de la hipertensión, diabetes, obesidad, dislipidemia y el consumo de tabaco generó la búsqueda de alternativas para enfrentar dos frentes al mismo tiempo: la detección oportuna y adecuada estratificación del riesgo y aumentar la porcentaje de control de los factores de riesgo cardiovascular a largo plazo. ${ }^{10}$ Recientemente se publicó un fascículo especial en una revista centrado en el Consenso Nacional de la Hipertensión Arterial en México y la situación clínica específica. ${ }^{11}$

\section{Modelo preventivo}

El Instituto Mexicano del Seguro Social ajustó su modelo de cuidado de curativo a preventivo, para lidiar con el crecimiento de las cuatro principales enfermedades crónicas degenerativas (diabetes, dolencias cardiovasculares, cáncer de mama y próstata), que constituyen las principales causas de la muerte en el país..$^{12-14}$ El nuevo modelo es desplegar brigadas médicas en los lugares de trabajo y crear nuevas clínicas en medicina especializada, con el apoyo de una unidad de inteligencia que se enfocará en el seguimiento mediante una placa electrónica y datos personalizados a tiempo, para detectar los riesgos potenciales de los derechohabientes en relación con estas enfermedades. El desarrollo de centros de 
control metabólico ambulatorio con unidades de inteligencia artificial representa un gran avance en esta institución.

\section{Futuro}

La prevención es una pieza angular en el cuidado del paciente en riesgo de desarrollar una enfermedad y para evitar complicaciones en quienes ya son portadores de la misma. No es una novedad que la hipertensión arterial sistémica constituye uno de los principales factores de riesgo cardiovascular. Es necesario tener en cuenta que la enfermedad es continua y que si bien los riesgos comienzan a partir de una presión arterial de 120/80 mm $\mathrm{Hg}$, cada caso debe individualizarse y estratificarse correctamente, para establecer medidas preventivas como primer paso. En todos los pacientes con hipertensión deben establecerse cambios en los hábitos alimentarios y de actividad física, a menos que estén contraindicados. En el seguimiento será necesaria la generación de cohortes para conocer el impacto de las medidas farmacológicas.

\section{Bibliografía}

1. Whelton PK, Carey RM, Aronow WS, Casey DE Jr, Collins KJ, et al. 2017 ACC/AHA/AAPA/ABC/ACPM/AGS/APhA/ASH/ASPC/NMA/PCNA
Guideline for the prevention, detection, evaluation, and management of high blood pressure in adults: a report of the American College of Cardiology/American Heart Association Task Force on Clinical Practice Guidelines. Hypertension. 2018;71(6): e13-e115.

2. Instituto Nacional de Salud Pública. Ensanut 2012. Hipertensión arterial en adultos mexicanos: importancia de mejorar el diagnóstico oportuno y el control. Disponible en: https://ensanut.insp.mx/doctos/analiticos/ HypertensionArterialAdultos.pdf

3. Instituto Nacional de Salud Pública. Encuesta Nacional de Salud y Nutrición de Medio Camino 2016. México: INSP; 2016. Disponible en: https://www.gob.mx/salud/documentos/encuesta-nacional-de-salud-y-nutricion-de-medio-camino-2016

4. Velázquez-Monroy O, Rosas-Peralta M, Lara-Esqueda A, Pastelín-Hernández $\mathrm{G}$, Attie $\mathrm{F}$, et al. Hipertensión arterial en México: resultados de la ENSA 2000. Arch Cardiol Mex. 2002;72:71-84.

5. Wang Ji-Guang, Lisheng LA. Global impact of 2017 American College of Cardiology/American Heart Association Hypertension Guidelines: a perspective from China. Circulation. 2018;137:546-548

6. Kario K. Global impact of 2017 American Heart Association/American College of Cardiology Hypertension Guidelines: a perspective from Japan. Circulation. 2018;137:543-545

7. Wander GS, Ram CVS. Global impact of 2017 American Heart Association/American College of Cardiology Hypertension Guidelines: a perspective from India. Circulation. 2018;137:549-550

8. Schifrin E. Global impact of the 2017 American College of Cardiology/ American Heart Association Hypertension Guidelines: a perspective from Canada. Circulation. 2018;137:883-885

9. Rodríguez-Ramírez M, Simental-Mendía LE, González-Ortiz M, Martínez-Abundis E, Madero A, et al. Prevalence of prehypertension in Mexi$\mathrm{Co}$ and its association with hypomagnesemia. Am $\mathrm{J}$ Hypertension. 2015;28:1024-1030.

10. Jaffe MG, Young JD. The kaiser permanent Northern California story: improving hypertension control from $44 \%$ to $90 \%$ in 13 years (2000 to 2013). J Clin Hypertens. 2016;18:260-261.

11. Rosas-Peralta M, Palomo-Piñón S, Borrayo-Sánchez G, et al. Consenso de Hipertensión Arterial Sistémica en México. Rev Med Inst Mex Seguro Soc. 2016;54:6-51.

12. Borja-Aburto VH. Salud pública. México: Alfil; 2013.

13. Instituto Mexicano del Seguro Social. [Sitio web]. CHKT en línea. PREVENIMSS. Disponible en: http://www.imss.gob.mx/chkt

14. Castañeda-Sánchez O, Ruelas-Oliveros G. Efecto de una intervención educativa en preescolares con factores de riesgo cardiovascular en Ciudad Obregón, Sonora. Aten Fam. 2015;22:77-81. 